\title{
Antimicrobial Effects of Drugs against Multidrug-Resistant Pseudomonas aeruginosa
}

\author{
Kumiko Maeda, ${ }^{a}$ Youko Kobayashi, ${ }^{a}$ Shigeharu Oie, ${ }^{a}$ Shiro Ishida,,${ }^{b}$ Yoshiro Okano, ${ }^{b}$ \\ Toshihiko Kobayashi, ${ }^{c}$ Kyoko ShIKIChI, ${ }^{c}$ Hidekazu Mizuno, ${ }^{c}$ and Akira KamiYA $*, a$ \\ ${ }^{a}$ Department of Pharmacy, Yamaguchi University Hospital; ${ }^{c}$ Clinical Laboratory, Yamaguchi University Hospital; 1-1-1 \\ Minamikogushi, Ube 755-8505, Japan: and ${ }^{b}$ Department of Pharmaceutical Care and Clinical Pharmacy, Faculty of \\ Pharmaceutical Sciences, Tokushima Bunri University; 180 Yamahshiro-cho, Tokusima 770-8514, Japan. \\ Received March 28, 2008; accepted July 17, 2008
}

We evaluated the effects of antimicrobial drugs on four strains of Pseudomonas aeruginosa that are resistant to eight widely used antipseudomonal drugs (piperacillin, piperacillin-tazobactam, imipenem, meropenem, ceftazidime, aztreonam, amikacin, ciprofloxacin) and colistin. In the killing test, colistin $(2 \mu \mathrm{g} / \mathrm{ml})$ was the most effective, followed by aztreonam $(48 \mu \mathrm{g} / \mathrm{ml})$, piperacillin-tazobactam $(192-4 \mu \mathrm{g} / \mathrm{ml})$, piperacillin $(192 \mu \mathrm{g} / \mathrm{ml})$, and a three drug combination of azetreonam $(16 \mu \mathrm{g} / \mathrm{ml})$, ceftazidime $(16 \mu \mathrm{g} / \mathrm{ml})$, and amikacin $(4 \mu \mathrm{g} / \mathrm{ml})$. Six hours after drug addition, colistin $(2 \mu \mathrm{g} / \mathrm{ml})$, aztreonam $(48 \mu \mathrm{g} / \mathrm{ml})$, piperacillin-tazobactam $(192-4 \mu \mathrm{g} / \mathrm{ml})$, piperacillin $(192 \mu \mathrm{g} / \mathrm{ml})$, and the above three drug combination had bacteriostatic effects on all four strains. Colistin, three time breakpoint of aztreonam, piperacillin, or piperacillin-tazobactam, and the three drug combination of aztreonam, ceftazidime, and amikacin were effective in vitro.

Key words Pseudomonas aeruginosa; multidrug-resistant; aztreonam; ceftazidime; amikacin; combined effect

Multidrug-resistant gram-negative rods such as Pseudomonas aeruginosa, Acinetobacter baumannii, and Klebsiella pneumoniae have presented problems. ${ }^{1,2)}$ In particular, $P$. aeruginosa is an important species causing nosocomial infection, ${ }^{3)}$ and its development of multidrug-resistance has become a major social issue. ${ }^{4-6)}$

In Japan, multidrug-resistant $P$. aeruginosa was defined as isolates resistant to all of the following drugs: imipenem, MIC (minimum inhibitory concentration) $\geq 16 \mu \mathrm{g} / \mathrm{ml}$; amikacin, $\mathrm{MIC} \geq 32 \mu \mathrm{g} / \mathrm{ml}$; and ciprofloxacin, $\mathrm{MIC} \geq 4 \mu \mathrm{g}$ / $\mathrm{ml}$. However, some of these isolates resistant to imipenem, ceftazidime, and amikacin are also resistant to all other commonly used antipseudomonal drugs such as piperacillintazobactam, aztreonam, and ciprofloxacin. For such isolates, drugs for treatment are not available at present. Our previous studies have shown that three drug combinations such as the combination of ceftazidime, aztreonam, and amikacin are more effective against multidrug-resistant $P$. aeruginosa than the combinations of $\beta$-lactam and aminoglycoside antibiotics, such as ceftazidime and amikacin. ${ }^{7,8)}$ Therefore, in this study, we evaluated the effects of three drug combinations of ceftazidime, aztreonam, and amikacin and ceftazidime, aztreonam, amikacin, or colistin alone at a concentration three times the breakpoint concentration on four strains of $P$. aeruginosa that were isolated from patients in two hospitals in 2002-2006 and resistant to all commonly used antipseudomonal drugs.

\section{MATERIALS AND METHODS}

Bacterial Strains Of a total of $1720 P$. aeruginosa strains isolated from clinical materials in two hospitals 733 and 315 beds, respectively in Yamaguchi Prefecture between January 2002 and December 2006, four strains that were resistant to all of piperacillin, piperacillin-tazobactam, imipenem, meropenem, ceftazidime, aztreonam, amikacin, and ciprofloxacin were used. The sources of the four strains were blood (two strains), bile (one), and frank hip pus (one).

Random Amplified Polymorphic DNA (Deoxyribonucleic Acid) Analysis ${ }^{9-11)}$ Genomic DNA was purified by the phenol-chloroform method. The primers 272 (5'-AGCGGGCCAA-3') and ERIC2 (enterobacterial repetitive intragenic consensus; 5'-ATGTAAGCTCCTGGGATTTCA-3') were employed. The reaction took place in $1 \mu \mathrm{l} 10 \times \mathrm{PCR}$ (polymerase chain reaction) buffer (Ex Taq, Takara, Tokyo, Japan), $0.8 \mu \mathrm{l}$ dNTP (deoxyribonucleoside triphosphates) mixture (Ex Taq, Takara, Tokyo, Japan), $0.06 \mu \mathrm{l}$ Ex Taq (Ex Taq, Takara, Tokyo, Japan), $0.2 \mu \mathrm{l}$ primer, $80 \mathrm{ng}$ DNA template, $6.94 \mu \mathrm{l}$ water, and $10 \mu \mathrm{l}$ mineral oil. Amplification was performed in a DNA thermal cycler (Perkin ELMER 9600) under the following conditions: primer $272 ; 94{ }^{\circ} \mathrm{C}$ for $7 \mathrm{~min}$ and $94^{\circ} \mathrm{C}$ for $1 \mathrm{~min}, 35^{\circ} \mathrm{C}$ for $1 \mathrm{~min}, 72^{\circ} \mathrm{C}$ for $1 \mathrm{~min}(40 \mathrm{cy}-$ cles), and $72^{\circ} \mathrm{C}$ for $16 \mathrm{~min}$. Primer ERIC2 was used under the same conditions except for the following: the temperature was changed from 35 to $52^{\circ} \mathrm{C}$, and the number of cycles from 40 to 35 . Amplification products were resolved by electrophoresis in a 3\% agarose gel and were detected by staining with ethidium bromide. Banding patterns were subsequently analyzed.

Susceptibility Tests by Agar Dilution Methods MICs were determined after $18 \mathrm{~h}$ of incubation at $37^{\circ} \mathrm{C}$ by dilution on Sensitivity Disc Agar-N (Nissui Pharmaceuticals, Tokyo, Japan). The following nine antimicrobial agents were tested: piperacillin (Toyama Chemicals, Tokyo, Japan), piperacillintazobactam (Toyama Chemicals, Tokyo, Japan), imipenem (Banyu Pharmaceuticals, Tokyo, Japan), meropenem (Dainippon Sumitomo Pharmaceuticals, Tokyo, Japan), ceftazidime (Glaxo Japan Co., Tokyo, Japan), aztreonam (Eisai Co., Tokyo, Japan), amikacin (Banyu Pharmaceuticals, Tokyo, Japan), ciprofloxacin (Bayer Japan Co., Tokyo, Japan), and colistin (Wako Chemical Co., Tokyo, Japan). These agents except for ciprofloxacin were provided in the form of a freeze-dried, amorphous powder. The inocula ( $\mathrm{ca} .10^{4} \mathrm{cfu}$ (colony-forming units)/spot) were plated using a multipoint 
inoculator (Sakuma Co., Tokyo, Japan). The MIC was defined as the lowest drug concentration that inhibited visible growth. $P$. aeruginosa IFO 3919 was used as a reference strain.

Drug Effects by Killing Tests Killing experiments were carried out to evaluate bactericidal activities. The drugs used were: piperacillin, piperacillin-tazobactam, meropenem, ceftazidime, aztreonam, amikacin, ciprofloxacin, colistin, and ceftazidime+azetreonam+amikacin (three drug combination). The concentrations of these drugs were the following breakpoints (breakpoints defining susceptibility or resistance of bacteria to antimicrobial agents) and concentrations three times the breakpoints: piperacillin, $64 \mu \mathrm{g} / \mathrm{ml}$; piperacillintazobactam, 64 and $4 \mu \mathrm{g} / \mathrm{ml}$, respectively; meropenem, $8 \mu \mathrm{g} /$ $\mathrm{ml}$; ceftazidime, $16 \mu \mathrm{g} / \mathrm{ml}$; aztreonam, $16 \mu \mathrm{g} / \mathrm{ml}$; amikacin, $4 \mu \mathrm{g} / \mathrm{ml}$; ciprofloxacin, $2 \mu \mathrm{g} / \mathrm{ml}$; and colistin, $2 \mu \mathrm{g} / \mathrm{ml})$. However, in the three drug combination (ceftazidime+aztreonam+ amikacin), the breakpoint concentrations of each drug were used. Breakpoints used for all agents tested except for amikacin and colistin were according to National Committee for Clinical Laboratory Standards (NCCLS) criteria. ${ }^{12)}$ The concentration of amikacin was $4 \mu \mathrm{g} / \mathrm{ml}$, which is lower than in the NCCLS criteria. This was because, in Japan, the routine dose of amikacin is lower $(200-400 \mathrm{mg} / \mathrm{d}$ in one to two divided doses) than that in Western countries. The breakpoint used for colistin was according to the Comité de l'Antibiogramme of the Société Française de Mirobiologie. ${ }^{13)}$

Sensitivity Test Broth (Nissui Pharm, Tokyo, Japan) was used as the medium. The final concentration of the log-phase inocula was approximately $5 \times 10^{5} \mathrm{cfu} / \mathrm{ml}^{7,14-16)}$ Viability was determined from bacterial counts carried out at 2, 4, 6, and $24 \mathrm{~h}$ after incubation at $37^{\circ} \mathrm{C}$ by plating $500 \mu \mathrm{l}$ of serial dilutions from each tube onto trypticase soy agar plates. The numbers of viable bacteria were counted after 24 and $48 \mathrm{~h}$ of incubation of the plates at $37^{\circ} \mathrm{C}$. In preliminary experiments, drug carryover was ruled out by plating samples of a bacterial suspension containing $c a .10^{2} \mathrm{cfu} / \mathrm{ml}$ in the presence or absence of antimicrobial agents alone or in combination. Bactericidal activity was defined as a $\geq 3 \log _{10} \mathrm{cfu} / \mathrm{ml}$ decrease in the starting inoculum. A bacteriostatic effect was defined as any decrease in the viable count from the starting inoculum. ${ }^{14)}$ This experiment was performed three times.

\section{RESULTS}

Of a total of 1720 P. aeruginosa strains clinically isolated in two hospitals 733 and 315 beds, respectively in Yamaguchi Prefecture between January 2002 and December 2006, four strains $(0.2 \%)$ were resistant to all the eight commonly used antipseudomonal drugs. The DNA pattern generated by random amplified polymorphic DNA analysis differed among the four strains. Table 1 shows the MICs of the eight commonly used antipseudomonal drugs and colistin for the four strains.

Figure 1 shows the mean killing curves for the four strains of multidrug-resistant $P$. aeruginosa in broth containing each drug at its breakpoint concentration. Seven drugs other than colistin had no bacteriostatic effects. However, colistin exhibited bacteriostatic effects until $6 \mathrm{~h}$ after its addition. Figure 2 shows the mean killing curves for the four strains of multidrug-resistant $P$. aeruginosa in broth containing each
Table 1. MICs $(\mu \mathrm{g} / \mathrm{ml})$ of Nine Antipseudomonal Agents against Four Strains of Multidrug-Resistant Pseudomonas aeruginosa

\begin{tabular}{lrrrr}
\hline \hline \multirow{2}{*}{ Agents } & \multicolumn{4}{c}{ MIC $(\mu \mathrm{g} / \mathrm{ml})$ for strains } \\
\cline { 2 - 5 } & \multicolumn{1}{c}{$\mathrm{a}$} & $\mathrm{b}$ & $\mathrm{c}$ & $\mathrm{d}$ \\
\hline Piperacillin & 128 & 128 & 128 & $>256$ \\
Piperacillin- $_{\quad \text { tazobactam }}^{a)}$ & 128 & 128 & 128 & $>256$ \\
Imipenem $_{\text {Meropenem }}$ & 128 & 128 & 64 & 64 \\
Ceftazidime & $>256$ & 128 & 32 & 64 \\
Aztreonam & 256 & 256 & 32 & 128 \\
Amikacin & 32 & 32 & 64 & 128 \\
Ciprofloxacin & 256 & 128 & $>256$ & $>256$ \\
Colistin & 256 & $>256$ & 16 & 8 \\
& 1 & 1 & 2 & $<0.25$ \\
\hline
\end{tabular}

a) This agent is a mixture of piperacillin and tazobactam. Concentrations are expressed as those of piperacillin (the tazobactam concentration was fixed at $4 \mu \mathrm{g} / \mathrm{ml}$ ).

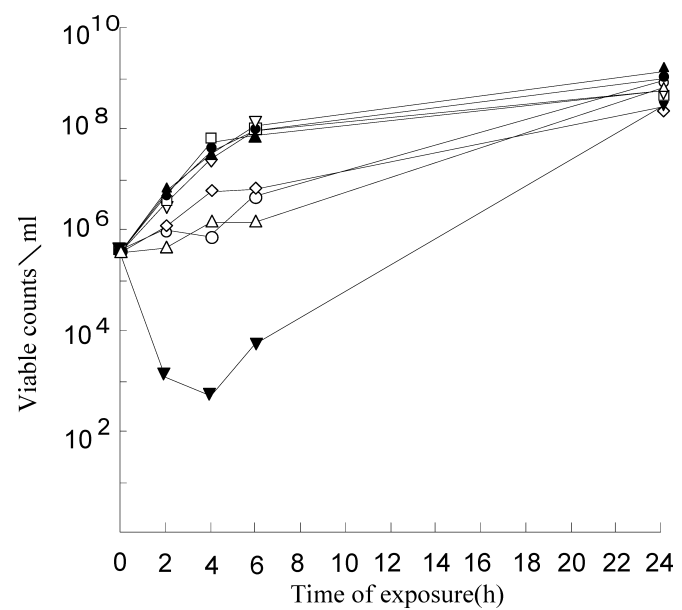

Fig. 1. Mean Killing Curves of Multidrug-Resistant Pseudomonas aeruginosa* in Broth Containing $64 \mu \mathrm{g} / \mathrm{ml}$ Piperacillin $(\bigcirc), 64 \mu \mathrm{g} / \mathrm{ml}$ Piperacillin with $4 \mu \mathrm{g} / \mathrm{ml}$ Tazobactam $(\triangle), 8 \mu \mathrm{g} / \mathrm{ml}$ Meropenem $(\nabla), 16 \mu \mathrm{g} / \mathrm{ml}$ Ceftazidime $(\square), 16 \mu \mathrm{g} / \mathrm{ml}$ Aztreonam $(\diamond), 4 \mu \mathrm{g} / \mathrm{ml}$ Amikacin $(\bigcirc), 2 \mu \mathrm{g} / \mathrm{ml}$ Ciprofloxacin $(\boldsymbol{\Delta})$, and $2 \mu \mathrm{g} / \mathrm{ml}$ Colistin ( $)$

* Four strains were evaluated.

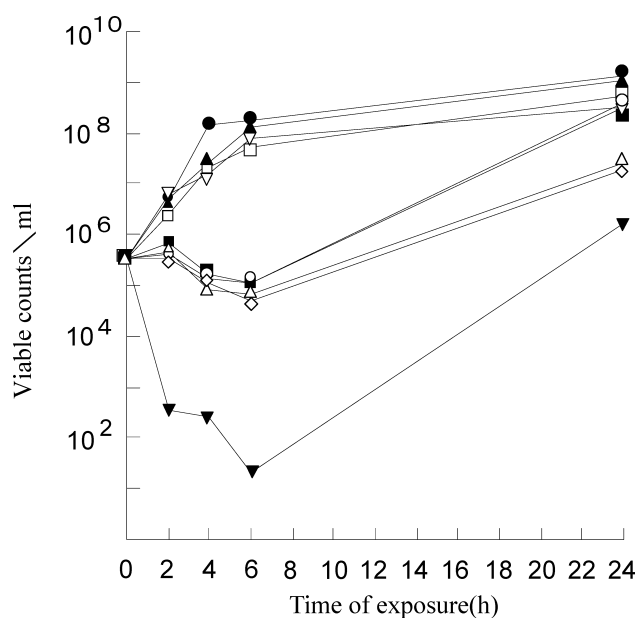

Fig. 2. Mean Killing Curves of Multidrug-Resistant Pseudomonas aeruginosa $^{*}$ in Broth Containing $192 \mu \mathrm{g} / \mathrm{ml}$ Piperacillin (O), $192 \mu \mathrm{g} / \mathrm{ml}$ Piperacillin with $4 \mu \mathrm{g} / \mathrm{ml}$ Tazobactam $(\triangle), 24 \mu \mathrm{g} / \mathrm{ml}$ Meropenem $(\nabla)$, $48 \mu \mathrm{g} / \mathrm{ml}$ Ceftazidime $(\square), 48 \mu \mathrm{g} / \mathrm{ml}$ Aztreonam $(\diamond), 12 \mu \mathrm{g} / \mathrm{ml}$ Amikacin

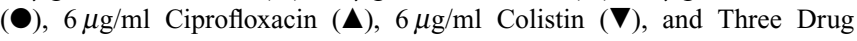
Combination of $16 \mu \mathrm{g} / \mathrm{ml}$ Ceftazidime $+16 \mu \mathrm{g} / \mathrm{ml}$ Aztreonam $+4 \mu \mathrm{g} / \mathrm{ml}$ Amikacin (

* Four strains were evaluated. 
Table 2. Effects of Antipseudomonal Agents on Four Strains of Multidrug-Resistant Pseudomonas aeruginosa (Killing Test)

\begin{tabular}{|c|c|c|c|c|c|c|c|c|c|}
\hline \multirow{3}{*}{ Agent } & \multirow{3}{*}{$\begin{array}{l}\text { Concentration } \\
(\mu \mathrm{g} / \mathrm{ml})\end{array}$} & \multicolumn{8}{|c|}{ Strains showing effects $\left.{ }^{a}\right)$} \\
\hline & & \multicolumn{4}{|c|}{ Bacteriostatic effects } & \multicolumn{4}{|c|}{ Bactericidal effects } \\
\hline & & $2 h^{b)}$ & $4 \mathrm{~h}$ & $6 \mathrm{~h}$ & $24 \mathrm{~h}$ & $2 \mathrm{~h}$ & $4 \mathrm{~h}$ & $6 \mathrm{~h}$ & $24 \mathrm{~h}$ \\
\hline Piperacillin & 64 & $\mathrm{c}$ & $\mathrm{b}, \mathrm{c}$ & $\mathrm{b}$ & & & & & \\
\hline Piperacillin-tazobactam & $64 / 4$ & $\mathrm{~b}, \mathrm{c}$ & $\mathrm{a}, \mathrm{b}, \mathrm{c}$ & $b, c$ & & & & & \\
\hline Meropenem & 8 & $\mathrm{c}$ & $\mathrm{c}$ & $\mathrm{c}$ & & & & & \\
\hline Ceftazidime & 16 & & $\mathrm{c}, \mathrm{d}$ & $c, d$ & & & & & \\
\hline Aztreonam & 16 & & $\mathrm{~b}$ & $\mathrm{~b}$ & & & & & \\
\hline Amikacin & 4 & & & & & & & & \\
\hline Ciprofloxacin & 2 & & & & & & & & \\
\hline Colistin & 2 & All & All & All & $\mathrm{c}, \mathrm{d}$ & & $\mathrm{b}, \mathrm{c}, \mathrm{d}$ & d & d \\
\hline Piperacillin & 192 & $b, d$ & $b, c, d$ & All & & & & & \\
\hline Piperacillin-tazobactam & $192 / 4$ & $b, d$ & All & All & & & & & \\
\hline Meropenem & 24 & $\mathrm{~b}, \mathrm{c}$ & c & $\mathrm{c}$ & $\mathrm{c}$ & & & & \\
\hline Ceftazidime & 48 & $c, d$ & $\mathrm{c}, \mathrm{d}$ & $c, d$ & $\mathrm{c}$ & & & & \\
\hline Aztreonam & 48 & $b, c, d$ & $b, c, d$ & All & & & & & \\
\hline Amikacin & 12 & & & & & & & & \\
\hline Ciprofloxacin & 6 & & $\mathrm{~d}$ & $\mathrm{~d}$ & & & & & \\
\hline Colistin & 6 & All & All & All & $b, c, d$ & $\mathrm{a}, \mathrm{b}, \mathrm{d}$ & $a, b, d$ & All & $b, d$ \\
\hline $\begin{array}{l}\text { Ceftazidime } \\
\text { +aztreonam + amikacin }\end{array}$ & $\begin{array}{l}\text { Ceftazidime } \\
\text { and aztreonam, } \\
16 \mu \mathrm{g} / \mathrm{ml} \text { each; } \\
\text { amikacin, } 4 \mu \mathrm{g} / \mathrm{ml}\end{array}$ & d & All & All & d & & d & d & d \\
\hline
\end{tabular}

a) Bacteriostatic effects, any decrease in the viable count from the starting inoculum; bactericidal effects, $\geq 3 \log { }_{10}$ cfu/ml decrease in the starting inoculum. $\quad b$ ) Time after drug addition

drug at a concentration three times its breakpoint or three combined drugs (ceftazidime + aztreonam + amikacin) at their breakpoint concentrations. Meropenem, ceftazidime, amikacin and ciprofloxacin showed no bacteriostatic effects within $6 \mathrm{~h}$ after addition. However, piperacillin, piperacillin-tazobactam, aztreonam, and the three combined drugs (ceftazidime+aztreonam + amikacin) exerted bacteriostatic effects. Colistin showed not only bacteriostatic but also bactericidal effects. This experiment was performed three times, and the result was reproducible.

Table 2 shows the effects of antimicrobial drugs on the four strains of multidrug-resistant $P$. aeruginosa (killing test). Within $6 \mathrm{~h}$ after the addition of each drug at its breakpoint concentration, amikacin and ciprofloxacin had no bacteriostatic effects on any of the four strains, while piperacillin, piperacillin-tazobactam, meropenem, ceftazidime, and aztreonam exhibited bacteriostatic effects on $1-3$ strains. Colistin showed bacteriostatic effects on all four strains. On the other hand, within $6 \mathrm{~h}$ after the addition of each drug at a concentration three times its breakpoint concentration, meropenem, ceftazidime, amikacin, and ciprofloxacin exerted bacteriostatic effects on $0-2$ strains, while piperacillin, piperacillintazobactam, aztreonam, and colistin exerted bacteriostatic effects on all four strains. The three drug combination (ceftazidime + aztreonam + amikacin at their breakpoint concentrations) had bacteriostatic effects on all four strains within $6 \mathrm{~h}$ after addition. Within $6 \mathrm{~h}$ post-addition, colistin showed bactericidal effects on three of the four strains at its breakpoint concentration and on all four strains at a concentration three times the breakpoint concentration.

\section{DISCUSSION}

The detection rate of clinical isolates of $P$. aeruginosa showing resistance to all commonly used antipseudomonal drugs is low, but is expected to increase in the future. Even the two drug combination of $\beta$-lactam antibiotics and aminoglycoside antibiotics has inadequate antimicrobial effects on such $P$. aeruginosa strains. ${ }^{7,8)}$

We evaluated the antimicrobial effects of various drugs including a three drug combination and colistin, which is not commercially available in Japan, on P. aeruginosa strains resistant to all commonly employed antipseudomonal agents. The three drug combination (ceftazidime+aztreonam + amikacin) and piperacillin $(192 \mu \mathrm{g} / \mathrm{ml})$, piperacillin-tazobactam $(192-4 \mu \mathrm{g} / \mathrm{ml})$, and aztreonam $(48 \mu \mathrm{g} / \mathrm{ml})$ exhibited bacteriostatic effects on all four strains of multidrugresistant $P$. aeruginosa. The three drug combination (ceftazidime+aztreonam+amikacin) may be an effective treatment method against infection with $P$. aeruginosa resistant to all commonly used drugs. Piperacillin $(192 \mu \mathrm{g} / \mathrm{ml})$, piperacillin-tazobactam $(192-4 \mu \mathrm{g} / \mathrm{ml})$, and aztreonam $(48 \mu \mathrm{g} / \mathrm{ml})$ also had bacteriostatic effects on the four strain of multidrug-resistant $P$. aeruginosa. These drugs are negligibly toxic and can be administered at high doses. After high-dose administration, a blood concentration thee times the breakpoint can be achieved. ${ }^{17-19)}$ Therefore, high-dose administration of piperacillin, piperacillin-tazobactam, or aztreonam should be also considered for infection with $P$. aeruginosa resistant to all commonly used drugs.

Among the tested drugs, colistin showed the most marked antimicrobial effects on multidrug-resistant $P$. aeruginosa. Colistin had severe adverse effects such as kidney disor- 
ders, ${ }^{20,21)}$ but should be selected when three drug administration (ceftazidime, aztreonam+amikacin) or high-dose administration of aztreonam is ineffective. Some previous studies have revealed the effects of colistin on multidrug-resistant $P$. aeruginosa infection. ${ }^{22-24)}$ In addition, some studies have shown that the adverse effects of colistin are less severe than those conventionally considered. ${ }^{24-26)}$

\section{REFERENCES}

1) Landman D., Bratu S., Kochar S., Panwar M., Trehan M., Doymaz M., Quale J., J. Antimicrob. Chemother., 60, 78-82 (2007).

2) Karlowsky J. A., Draghi D. C., Jones M. E., Thornsberry C., Friedland I. R., Sahm D. F., Antimicrob. Agents Chemother., 47, 1681-1688 (2003).

3) Rolston K. V., Bodey G. P., Cancer Invest., 10, 43-59 (1992).

4) the NNIS System, Am. J. Infect. Control, 27, 520-532 (1999).

5) Bonfiglio G., Carciotto V., Russo G., Stefani S., Schito G. C., Debbia E., Nicoletti G., J. Antimicrob. Chemother, 41, 307-310 (1998).

6) Livermore D. M., Antimicrobial Resistance, 34, 634-640 (2002).

7) Oie S., Uematsu T., Sawa A., Mizuno H., Tomita M., Ishida S., Okano Y., Kamiya A., J. Antimicrob. Chemother, 52, $911-914$ (2003).

8) Oie S., Sawa A., Kamiya A., Mizuno H., J. Antimicrob. Chemother, 44, 689-691 (1999).

9) Nihonyanagi S., Hirata Y., Akabosi T., Uchiyama Y., Yamaura N., Sunakawa K., Inoue M., J. Jpn. Assoc. Infect. Dis., 80, 97-102 (2006).

10) Mahenthiralingam E., Campbell M. E., Foster J., Lam J. S., Speert D. P., J. Clin. Microbiol., 34, 1129-1135 (1996).

11) Barnini S., Dodi C., Campa M., Lett. Appl. Microbiol., 39, 274-277 (2004).

12) National Commitee for Clinical Laboratory Standards, "Methods for Dilution Antimicrbial Susceptibility Tests for Bacteria that Grow Aer-
obically-Second Edition," Approved Standard M7-A2. NCCLS, Villanova, PA, 1990.

13) Soussy C. J., Cluzel R., Courvalin P. E. J., Clin. Microbiol. Infect. Dis., 13, 238-246 (1994).

14) Rochon-Edouard S., Pestel-Caron M., Lemeland J. F., Caron F., Antimicrob. Agents Chemother, 44, 3055-3060 (2000).

15) Giamarellos-Bourboulis E. J., Grecka P., Giamarellou H., J. Antimicrob. Chemother, 38, 287-291 (1996).

16) Hallander H. O., Dornbusch K., Gezelius L., Jacobson K., Karlsson I., Antimicrob. Agents Chemother, 22, 743 -752 (1982).

17) Nakagawa K., Yasuda Y., Nakajima Y., Watanabe Y., Noguchi M., Suzuki T., Jpn. J. Antibiot., 12, 2797-2804 (1982).

18) Matsumoto T., Nagatake T., Oishi K., Amamoto T., Urae R., Irie S., Nii Y., Urae A., J. Infect. Chemother, 42, 281-299 (1994).

19) Ohara K., Yamamoto K., Mogi H., Ito T., Saito M., Ono Y., Nagata K., Kamiya O., Hoshino A., Hirano M., Maruyama F., Ohno R., Adachi K., Kitaori K., Shirakawa S., Minami N., Katayama N., Tanaka M., Kobayashi M., Ikeda Y., Kodera Y., Matsushita T., Mitomo Y., Nitta M., Kodama H., Yamada K., Naito K., J. Infect. Chemother, 37, 1062-1070 (1989).

20) Evans M. E., Feola D. J., Rapp R. P., Ann. Pharmacother, 33, 960967 (1999).

21) Levin A. S., Barone A. A., Penço J., Santos M. V., Marinho I. S., Arruda E. A., Manrique E. I., Costa S. F., Clin. Infect. Dis., 28, 10081011 (1999).

22) Berlana D., Llop J. M., Fort E., Badia M. B., Jódar R., Am. J. HealthSyst. Pharm., 62, 39-47 (2005).

23) Linden P. K., Kusne S., Coley K., Fontes P., Kramer D. J., Paterson D., Clin. Infect. Dis., 37, 154-160 (2003).

24) Markou N., Apostolakos H., Koumoudiou C., Athanasiou M., Koutsoukou A., Alamanos I., Gregorakos L., Crit. Care, 7, 78-83 (2003).

25) Falagas M. E., Kasiakou S. K., Crit. Care, 10, 1-13 (2006).

26) Kallel H., Bahloul M., Hergafi L., Akrout M., Ketata W., Chelly H., Hamida C. B., Rekik N., Hammami A., Bouaziz M., Int. J. Antimicrob. Agents, 28, 366-369 (2006). 